\title{
An Optimal Shape Design Problem for Fan Noise Reduction
}

\section{Bahram Farhadinia}

Department of Math, University of Mohaghegh Ardabili, Ardabil, Iran.

Email: farhadinia@uma.ac.ir

Received December 24 ${ }^{\text {th }}, 2009$; revised April 10 $0^{\text {th }}, 2010$; accepted April 12 $2^{\text {th }}, 2010$.

\begin{abstract}
The objective of the present article is to find an optimal design of a fan inlet to reduce the amount of noise radiated to the far field from the system. Against the gradient-based optimization algorithms, we employ here a method based on measure theory which does not require any information of gradients and the differentiability of cost function.
\end{abstract}

Keywords: Shape Optimization, Noise Radiation, Measure Theory, Linear Programming

\section{Introduction}

Noise generated by the rotating blades of an aircraft turbofan engines continues to pose a challenge for many researchers and of course, the reduction of the noise have been received more attention in the recent studies. Williams and Hall studied aerodynamic sound generation by turbulent flow around a scattering half plane [1]. Howe in [2] discussed the trailing edge noise at low Mach numbers. It is well known that the aircraft noise may be minimized through the two signification tasks: acoustic shape optimization of the inlet [3] and impedance optimization of the liner [4]. In much of the pervious work on this topic, the computational methods are gradient-based that bear some drawbacks such as mesh regularization and the requirement of knowledge of derivatives.

The aim of this article is to present a method to find an optimal solution of a shape design problem that models reducing the amount of noise radiated from aircraft turbofan engines.

Our method is based on measure theory and has some features, for instance, the design process is not iterative and therefore requires no initial design to be suggested; it is computationally efficient and flexible enough to accommodate general design problem.

The paper proceeds as follows. In Section 2 we first describe the mathematical formulation of the optimal control problem and then a brief review of the weak formulation of the problem is given. In Section 3, we metamorphose the optimization problem to a linear programming problem by the aid of our method. Finally, we present some numerical results in Section 4.

\section{Problem Description and Weak Formulation}

We follow here the same set up as considered in [4] in where the geometry of the axisymmetric fan is as shown in Figure 1. We suppose that the model composition of the aircraft noise source is specified on the source plane $\Gamma_{f}$. The inlet of fan is surrounded by two boundaries: fixed boundary $\Gamma_{c}$ and flexible boundary $\Gamma_{\alpha}$ which is characterized by the function $\mathrm{y}=\alpha(x)$. It is assumed an acoustic liner exists on the boundary $\Gamma_{c}$. We let $\Gamma_{\infty}$ be enough far from the noise source. This implies that the radiated field treats locally as a plane wave at local incidence and in this case the Sommerfeld radiation boundary condition is satisfied. If we describe the treatment of the acoustic velocity potential $u$ with the Helmholtz equation as

$$
\Delta u+k^{2} u=0, \quad \text { on } \Omega,
$$

Subject to the boundary conditions

$$
\begin{aligned}
& \left.u\right|_{\Gamma_{f}}=g(\alpha), \\
& \left.\frac{\partial u}{\partial n}\right|_{\Gamma_{\alpha}}=0, \\
& \left.\frac{\partial u}{\partial n}\right|_{\Gamma_{a}}=0, \\
& \left.\left(u+x \frac{\partial u}{\partial n}\right)\right|_{\Gamma_{c}}=0, \\
& \left.\left(i k u+x \frac{\partial u}{\partial n}\right)\right|_{\Gamma_{\infty}}=0,
\end{aligned}
$$




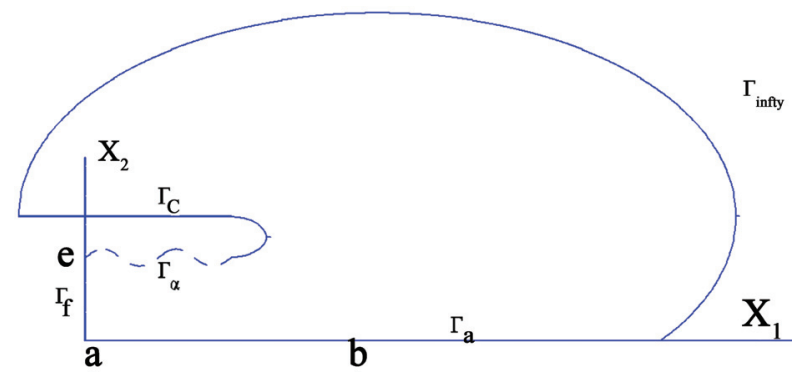

Figure 1. Geometry of the axisymmetric fan

then, the problem here is the manipulation of the flexible boundary $\Gamma_{\alpha}$ such that the least amount of noise propagates to the far field whereas some constraints on the boundary shape are satisfied. It is supposed that in the above equations, both the dependent and the independent variables are properly nondimensionalized.

Under consideration in [4], the optimal shape design problem may be stated as

$$
\begin{gathered}
\min \left\{J=A \int_{\Omega} \mathrm{u}^{2} d \Omega+B \int_{\Omega}|\nabla u|^{2} d \Omega+\lambda \int_{a}^{b}\left(\alpha\left(x_{1}\right)-\alpha_{0}\left(x_{1}\right)\right)^{2} d x_{1} ;\right. \\
\Delta u+k^{2} u=0, \quad \text { on } \quad \Omega, \\
\left.u\right|_{\Gamma_{f}}=g(\alpha), \\
\left.\frac{\partial u}{\partial n}\right|_{\Gamma_{\alpha}}=0 \\
\left.\frac{\partial u}{\partial n}\right|_{\Gamma_{a}}=0 \\
\left.\left(u+x \frac{\partial u}{\partial n}\right)\right|_{\Gamma_{c}}=0 \\
\left.\left.\left(i k u+x \frac{\partial u}{\partial n}\right)\right|_{\Gamma_{\infty}}=0\right\}
\end{gathered}
$$

where $x>0$ and the three constants A, B and $\lambda$ satisfy $A^{2}+B^{2}>0, \quad \lambda \geq 0$.

Obviously the determination of domain of the problem, $\Omega$, depends on the determination of $\alpha:[\mathrm{a}, \mathrm{b}] \rightarrow[\mathrm{c}, \mathrm{d}]$, which is a function to be determined by the optimization process. By virtue of this fact we let $\Omega=\Omega(\alpha)$.

In order for the optimal shape be exist, we define the admissible set of functions, $\alpha$ 's, as

$$
\mathrm{A}_{\mathrm{ad}}=\left\{\alpha \in \mathrm{C}([\mathrm{a}, \mathrm{b}]) ; \mathrm{c} \leq \alpha \leq \mathrm{d},\left|\alpha\left(\mathrm{t}_{1}\right)-\alpha\left(\mathrm{t}_{2}\right)\right| \leq \beta\left|\mathrm{t}_{1}-\mathrm{t}_{2}\right|\right\} .
$$

\section{Metamorphosis and Approximation}

If we define $\varphi=u-u_{0}$ and $V_{\alpha}=\left\{u \in H^{1}(\Omega(\alpha))\right.$; $\left.\left.u\right|_{\Gamma_{f}}=0\right\}$, then the variational formulation of $(P)$ with homogeneous boundary condition may be considered as (see [4])

$$
\begin{aligned}
& \left(p_{v}\right) \min \{J(\alpha, \phi)= \\
& A \int_{\Omega(\alpha)}\left|\phi+u_{0}\right|^{2} d \Omega+B \int_{\Omega(\alpha)}\left|\nabla\left(\phi+u_{0}\right)\right|^{2} d \Omega+\lambda \int_{a}^{b}\left(\alpha\left(x_{1}\right)-\alpha_{0}\left(x_{1}\right)\right)^{2} d x ; \\
& (\nabla \phi, \nabla v)-k^{2}(\phi, v)+<x \phi, v>\Gamma_{c}(\alpha)+i<\phi, v>\Gamma_{\infty}(\alpha)
\end{aligned}
$$

In this portion, we define an artificial control $w(x)_{1}:[a, b] \rightarrow R$ satisfying

$$
\begin{aligned}
& \frac{d}{d x_{1}} \alpha\left(x_{1}\right)=w\left(x_{1}\right), \forall x_{1} \in[a, b], \\
& \alpha(a)=\alpha(a)=e .
\end{aligned}
$$

In fact, $w$ is derivative of the boundary function $\Gamma_{\alpha}$. Let $x_{s}: S \rightarrow\{0,1\}$ be the characteristic function defined on the set $\mathrm{S}$.

We may now reformulate $\left(\mathrm{p}_{v}\right)$ involving the artificial control as follows:

$(\hat{P}) \min \left\{J(\alpha, \varphi)=\int_{\Omega(\alpha)}\left(A\left|\varphi+u_{0}\right|^{2}+B\left|\nabla\left(\varphi+u_{0}\right)\right|^{2}+\lambda x_{D}\right) d \Omega\right.$

$\int_{\Omega(\alpha)}\left(\nabla \varphi \bar{\nabla} \overline{\mathrm{v}}-\mathrm{k}^{2} \varphi \bar{v}-f \bar{v}\right) d \Omega+\int_{\partial \Omega(\alpha)}{ }^{x \varphi \bar{v} x} \Gamma_{c(\alpha)}{ }^{+i k \varphi \bar{v} x} \Gamma_{\infty(\alpha)} d \Gamma=0$, $\left.\forall \mathrm{v} \in \mathrm{V}_{\alpha} \frac{d}{d x_{1}} \alpha\left(x_{1}\right)=w\left(x_{1}\right), \forall x_{1} \in[a, b] \quad \alpha(a)=\alpha(a)=e\right\}$,

where $D_{1}=\left\{\left(x_{1}, x_{2}\right): x_{1} \in[a, b], 0 \leq x_{2} \leq\left(\alpha\left(x_{1}\right)-\alpha_{0}\left(x_{1}\right)\right)^{2}\right\}$.

In order to apply the metamorphosis process, one needs first to have some background knowledge.

We say a triple $q=(w, \alpha, \varphi)$ is admissible if the following conditions yield: (i) The artificial control $w$ takes its values in a compact set $\mathrm{W}$ and holds (2). (ii) The pair $(\alpha, \varphi)$ is the solution of the variational formulation of $\left(\mathrm{p}_{v}\right)$. We denote the set of all admissible triples by $Q$.

Let $\gamma_{1}=\hat{\Omega} \times S_{w} \times S_{\varphi} \times S_{\varphi^{\prime}} \times S_{\alpha}$ and $\gamma_{2}=\partial \hat{\Omega} \times S_{\varphi} \times S_{\alpha}$ where all these sets are compact and $w, \varphi, \nabla \varphi$ and $\alpha$ get their values in $S_{w}, S_{\varphi}, S_{\varphi^{\prime}}$ and $S_{\alpha}$, respectively. Also $\hat{\Omega}$ is the fixed domain depicted in Figure 2 and yields $\bigcup_{a \in A d a} \Omega(\alpha) \subset \hat{\Omega}$.

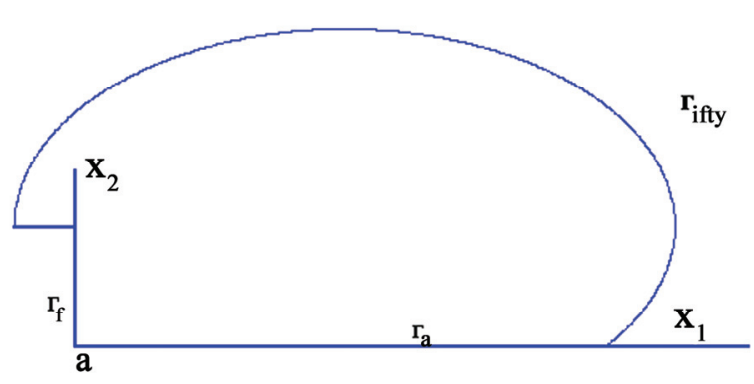

Figure 2. Geometry of the fixed domain $\hat{\Omega}$ 
Now we transfer $(\hat{\mathrm{P}})$ over $Q$, into another nonclassical problem that has some interesting properties.

Define two linear, bounded and positive functionals corresponding to each admissible triple $q$ as

$$
\begin{gathered}
\Lambda_{q, 1}: F \in C\left(\gamma_{1}\right) \rightarrow \int_{\Omega(\alpha)} F\left(x_{1}, x_{2}, w, \varphi, \nabla \varphi, \alpha\right) d \Omega,(3) \\
\Lambda_{q, 2}: G \in C\left(\gamma_{2}\right) \rightarrow \int_{\partial \Omega(\alpha)} G\left(x_{1}, x_{2}, \varphi, \alpha\right) d \Gamma
\end{gathered}
$$

where $C\left(\gamma_{i}\right)$ is the space of all continuous real-valued functions on $\gamma_{i}, i=1,2$.

By the use of the latter definition, one can observe that there exists an injection of $Q$ into $C^{*}\left(\gamma_{1}\right) \times C^{*}\left(\gamma_{2}\right)$, the product of dual spaces of functionals defined in (3)-(4). (See Proposition 4.1 in [5]).

By Riesz representation theorem [6], corresponding to each $\Lambda_{q, i}$, there is a unique positive Radon measure $\mu_{i}$, such that

$$
\Lambda_{q, I}(F)=\int_{\Omega(\alpha)} F\left(x_{1}, x_{2}, w, \varphi, \nabla \varphi, \alpha\right) d \Omega=\mu_{1}(F), \quad \forall F \in C\left(\gamma_{1}\right),
$$

$$
\Lambda_{q, 2}(G)=\int_{\partial \Omega(\alpha)} G\left(x_{1}, x_{2}, \varphi, \alpha\right) d \Gamma=\mu_{2}(G), \quad \forall G \in C\left(\gamma_{2}\right) .
$$

Generally, the metamorphosis process deals with integral form of relations, therefore, in order to convert the differential Equation (2) into an integral form, let $B$ be an open ball in $R^{2}$ containing $\hat{\Omega}$, and $C^{1}(B)$ be the space of all real-valued continuous differentiable functions with continuous first partial derivatives on $B$. Let $\psi \in C^{1}(B)$ and define

$$
\psi^{w}\left(x_{1}, x_{2}, w, \alpha\right)=\psi_{x_{2}}\left(x_{1}, \alpha\right) w\left(x_{1}\right)+\psi_{x 1}\left(x_{1}, \alpha\right)
$$

for each $\left(x_{1}, x_{2}, w, \alpha\right) \in \Omega_{1}=[a, b] \times[c, d] \times S_{w} \times S_{\alpha}$.

Thus for each admissible triple $q$ the system (2) is equivalent with

$$
\begin{aligned}
\int_{a}^{b} \psi^{w}\left(x_{1}, x_{2}, w, \alpha\right) d x_{1}= & \int_{a}^{b}\left(\psi_{x_{2}}\left(x_{1}, \alpha\right) w\left(x_{1}\right)+\psi_{x 1}\left(x_{1}, \alpha\right)\right) d x_{1} \\
& =\psi(b, \alpha(b))-\psi(a, \alpha(a))=\Delta \psi
\end{aligned}
$$

Now for simplifying the representation of optimal shape design problem, we set

$$
\begin{aligned}
& f_{0}(\alpha, \varphi)=A\left|\varphi+u_{0}\right|^{2}+B\left|\nabla\left(\varphi+u_{0}\right)\right|^{2}+\lambda x_{D_{1}}, \\
& F_{1}(\alpha, \varphi, v)=\nabla \varphi \bar{\nabla} \overline{\mathrm{v}}-k^{2} \varphi \overline{\mathrm{v}}-f \overline{\mathrm{v}}, \\
& F_{2}^{\psi}(\alpha, w)=\psi^{w} x_{D_{2}}, \\
& G_{1}(\alpha, \varphi, v)=x \varphi \overline{\mathrm{v}} x_{\Gamma_{c(\alpha)}}+i k \varphi \overline{\mathrm{v}} x_{\Gamma_{\infty}(\alpha)},
\end{aligned}
$$

where $D_{2}=\left\{\left(x_{1}, x_{2}\right): x_{1} \in[a, b], 0 \leq x_{2} \leq \psi^{w}\right\}$.

By virtue of (5)-(6) and the above arrangement, we may restate $(\hat{\mathrm{P}})$ by the following measure representation form:

$$
\begin{aligned}
\left(P_{M}\right) \min \{ & \mu_{1}\left(f_{0}\right) ; \\
& \mu_{1}\left(F_{1}\right)+\mu_{2}\left(G_{1}\right)=0, \quad \forall v \in V_{\alpha}, \\
& \left.\mu_{1}\left(F_{2}^{\psi}\right)=\Delta \psi, \quad \forall \psi \in C^{1}(B)\right\} .
\end{aligned}
$$

In what follows, we employ a two-phase approximation procedure that converts $\left(P_{M}\right)$ to a linear programming (LP) problem. The procedure for constructing a LP problem whose solution approximates the action of the optimal pair of measures of $\left(P_{M}\right)$ is much like as that given in [5]. In the first phase of approximation procedure, we intend to deal with a problem subject to a finite number of constraints. Following from Proposition 5.1 in [5], our intension is fulfilled. Approximating measures involved in $\left(P_{M}\right)$ by the unitary atomic measures (see Proposition 5.2 in [5]) is performed in continuation of the first phase of approximation procedure and leads to appear unknown supports. The second phase is devoted to approximate the unknown supports by specified points. This task is carried out in cooperation with Proposition 5.3 in [5].

Following the mentioned procedure, the resulting LP is as

$$
\begin{aligned}
(L P) \min & \left\{\sum_{k=1}^{N_{1}} \xi_{k}^{1} f_{0}\left(z_{1}^{k}\right) ;\right. \\
& \sum_{k=1}^{N_{1}} \xi_{k}^{1} F_{1}\left(z_{1}^{k}\right)+\sum_{k=1}^{N_{2}} \xi_{k}^{2} G_{1}\left(z_{2}^{k}\right)=0, \quad 1=1,2, \ldots, m_{1}, \\
& \sum_{k=1}^{N_{1}} \xi_{k}^{1} F_{2}^{\psi_{j}}\left(z_{1}^{k}\right)=\Delta \psi_{j}, \quad j=1,2, \ldots, m_{2}, \\
& \left.\xi_{k}^{i} \geq 0, \quad k=1,2, \ldots, N_{i}, \quad i=1,2\right\} .
\end{aligned}
$$

The process of extracting a piecewise constant control function $w$ form the optimal solution $\left\{\xi_{k}^{i} \geq 0, k=1,2\right.$, $\left.\ldots, N_{i}, \quad i=1,2\right\}$ of $(L P)$ is based on the analysis given in [7].

\section{Numerical Result}

The data for the parameters of the model are listed as:

$$
\begin{aligned}
& {[a, b]=[0,2.5], e=1, S_{w}=[-1,1], S_{\alpha}=[0.6,1.2],} \\
& k=8, A=B=\lambda=1, \\
& v_{1}=\sin \left(l x_{1}\right), 1=1,2, \ldots, m_{1}=4, \\
& \psi_{j}^{w}=x_{1}^{j}, j=1,2, \ldots, m_{2}=5, \\
& u_{0}\left(x_{1}\right)=0, \alpha_{0}\left(x_{1}\right)=1 .
\end{aligned}
$$

By using a code written in MATLAB 7.1 for solving $(L P)$, we obtained the optimal approximated $\Gamma_{\alpha}$ and its corresponding optimal artificial control $w$ depicted in Figure 3. 

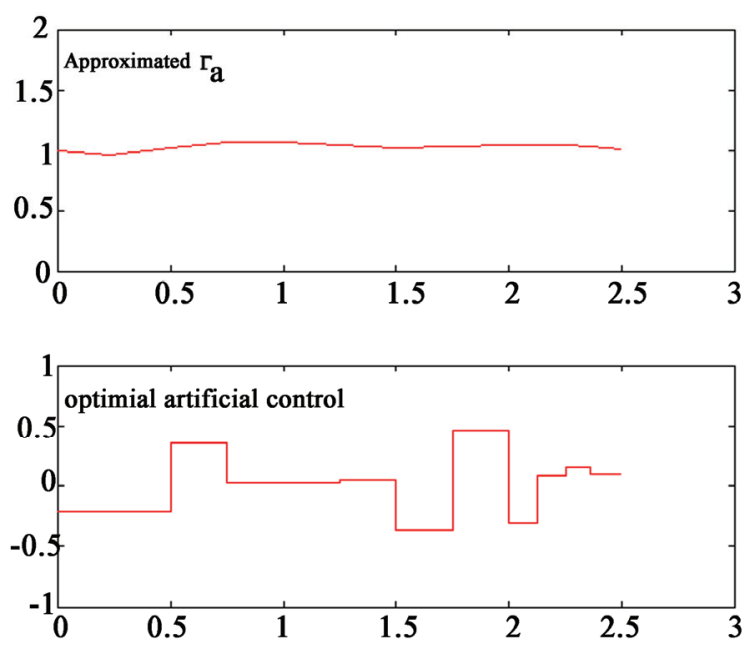

Figure 3. Optimal shape of the fan and optimal artificial control

\section{Conclusions}

This article discusses how to implement a numerical method based on measure theory for solving an optimal shape design problem for fan noise reduction. The proposed method has some advantages comparing to the gradient-based optimization methods. For instance, it does not require any information of gradients and the differentiability of cost function.

\section{REFERENCES}

[1] J. E. F. Williams and L. H. Hall, "Aerodynamic Sound Generation by Turbulent Flow in the Vicinity of a Scattering Half Plane," Journal of Fluid Mechanics, Vol. 40, No. 4, 1970, pp. 657-670.

[2] M. S. Howe, "Trailing Edge Noise at Low Mach Numbers," Journal of Sound and Vibration, Vol. 225, No. 2, 1999, pp. 211-238.

[3] A. L. Marsden, M. Wang and J. E. Dennis, "Constrained Aeroacoustic Shape Optimization Using the Surrogate Management Framework," Center for Turbulence Research, Annual Research Briefs, 2003.

[4] Y. Cao and D. Stanescu, "Shape Optimization for Noise Radiation Problems," Computers and Mathematics with Applications, Vol. 44, No. 12, 2002, pp. 1527-1537.

[5] B. Farhadinia, "Shape Optimization of a Nozzle with Specified Flow Field Including Viscosity Effect," Acta Applicandae Mathematicae, Vol. 104, No. 4, 2008, pp. 243-256.

[6] W. Rudin, "Real and Complex Analysis," McGraw-Hill, New York and London, 1970.

[7] J. E. Rubio, "Control and Optimization: The Linear Treatment of Nonlinear Problems," Manchester University Press, Manchester, 1986. 